\title{
Robot-Assisted Laparoscopic Versus Retropubic Radical Prostatectomy: Comparison of Functional and Oncological Outcomes, A Single-Center Experience
}

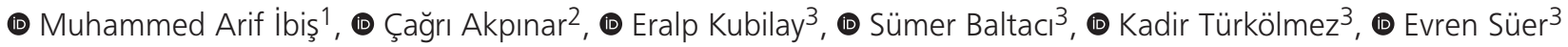 \\ ${ }^{1}$ Ankara Keçiören Training and Research Hospital, Clinic of Urology, Ankara, Turkey \\ ${ }^{2}$ Çubuk Halil Şıvgın State Hospital, Clinic of Urology, Ankara, Turkey \\ ${ }^{3}$ Ankara University Faculty of Medicine, Department of Urology, Ankara, Turkey
}

\begin{abstract}
Objective: This study aimed to investigate the effect of robot-assisted laparoscopic radical prostatectomy (RARP) and open retropubic radical prostatectomy (RRP) on perioperative functional and oncological outcomes.

Materials and Methods: This single-center retrospective study analyzed data of 443 patients who had undergone radical prostatectomy (RP) with localized prostate cancer. Surgical and clinicopathologic data, oncological and functional outcomes, and complications were compared between RRP and RARP groups. The comparison was made by the Mann-Whitney $\mathrm{U}$ test, chi-square test, and t-test for qualitative and quantitative variables, as appropriate. Log rank test was used to determine the biochemical recurrence-free survival of both surgical methods. Kaplan-Meier analysis was performed to estimate survival rates.

Results: The RRP and RARP groups included 231 and 212 patients, respectively. Blood loss, indwelling catheter duration, and hospitalization rates were low in the RARP group. Although the continence rates were better in the RARP group at 3 months, they were comparable at 12 months. In both groups, erection sufficient for sexual intercourse was comparable at 3 and 12 months. The mean lymph node yield was higher in the RRP group than in the RARP group. On median 28-month follow-up, no difference was found in the oncological results.

Conclusion: Although the oncological and functional results of RRP and RARP are comparable, RARP is a more minimally invasive procedure. In our opinion, the surgeon's experience is more effective than the chosen technique.
\end{abstract}

Keywords: Retropubic radical prostatectomy, prostate cancer, robotic prostatectomy, surgical technique

\section{Introduction}

Radical prostatectomy (RP), a curative treatment option for prostate cancer (PCa), was first described by Young in 1904 (1). After Young described the perineal approach, the retropubic approach, which is widely used today, was described by Millin in 1947 (2). Following the popularity of the dorsal vein complex and neurovascular bundle anatomy, the first nerve-sparing radical prostatectomy (NS-RP) was performed by Walsh in 1982 (3). Significant improvements in RP have been achieved in the last 30-40 years, and for the first time in 2001, this operation was performed by a robot-assisted laparoscopic approach (4).

$\mathrm{RP}$ is a treatment option widely used in clinically localized and locally advanced disease. The main purpose of RP is to remove the tumor tissue by preserving continence and potency. Currently, no specific data can show the superiority of any approach in terms of oncological and functional results after RP. Given the minimally invasive nature of robot-assisted laparoscopic radical prostatectomy (RARP), it may be associated with less blood loss and blood transfusion requirements, shorter hospitalization duration, and less pain.

In this study, we compared the preoperative, perioperative, and postoperative oncological and $3^{\text {rd }}$ month and $12^{\text {th }}$ month functional results of RARP and open retropubic radical prostatectomy (RRP).

\section{Materials and Methods}

\section{Patient Selection}

The study included patients who underwent RRP or RARP with a diagnosis of localized PCa between January 2016 and March 
2019, followed by at least 1 year, and whose data were fully available. The local ethical committee's registration number is 13-192-20. The study was conducted in a single center, and data of 443 patients were analyzed retrospectively. After a suspected digital rectal examination or a high prostatespecific antigen (PSA) value, diagnosis was made by transrectal ultrasound-guided prostate biopsy. If there are indications, bone scintigraphy, computed tomography, and multiparametric magnetic resonance imaging were performed. After the diagnosis was made, treatment options such as active surveillance, radiotherapy (RT), and RP were explained to the patients. The life expectancy of the patients was evaluated by the Charlson comorbidity index, and the risks of anesthesia were evaluated by using the American Society of Anesthesiologists score. The operation was planned for patients who had a life expectancy of at least 10 years and preferred RP as a treatment. Patients were informed about RRP and RARP by the surgeons who would perform the surgery. Patients decided whether the surgical approach was RRP or RARP. RP was performed at least 6 weeks after prostate biopsy to reduce possible surgical complications. On the night before the operation, enema as bowel preparation was performed and compression stockings were applied for all patients. Some of the RARP cases were performed by surgeons who were in the early phase of the learning curve. During the operation, extended lymph node (LN) dissection was performed in patients whose preoperative positive $\mathrm{LN}$ rate was more than $5 \%$. Nerve-sparing surgery was not performed on patients with a high risk of extracapsular diseases, such as any cT3a or cT3b PCa or any International Society of Urologic Pathology (ISUP) grade $>3$ on biopsy. Penile rehabilitation with phosphodiesterase-5 inhibitors was recommended to patients with sufficient erection capacity for sexual intercourse in the preoperative period, after the indwelling catheter was withdrawn in the postoperative period. Patients who had received RT as definitive therapy and then underwent salvage RP and those who performed transurethral prostatectomy before RP were excluded. The patients were divided into the RRP and RARP groups to compare selected parameters. The preoperative characteristics of the patients are shown in Table 1.

\section{Preoperative Parameters}

Age, body mass index, inguinal hernia or abdominal surgery history, potency and continence conditions, PSA value, biopsy ISUP grade, and clinical T stages were recorded.

\section{Intraoperative and Pathological Parameters}

Nerve-sparing LN dissection and intraoperative blood loss were recorded. NS-RP surgery was not performed on patients at high risk of extracapsular disease. Pathology specimens were evaluated by a single pathologist. Surgical margin positivity, pathology ISUP grade, and T stage were recorded in the examined pathology samples.

\section{Postoperative Parameters}

The postoperative parameters of the patients, such as hospitalization and catheterization time, and biochemical recurrence $(B C R)$ were examined. In follow-up measurements, PSA $>0.2 \mathrm{ng} / \mathrm{mL}$ in two consecutive measurements were accepted as BCR (5). Functional results, such as continence and erectile function, were questioned during the outpatient clinic controls performed at 3 and 12 months. Patients using more than one protective pad were considered incontinent, and patients who were unable to achieve a sufficient erection in more than half of their sexual intercourse were considered impotent. Patients using 2-3 protective pads daily were considered to have mild incontinence, and patients using more than 3 protective pads were considered to have serious incontinence.

\section{Statistical Analysis}

IBM SPSS ${ }^{\circledR}$ Statistics version 25 was used for statistical analysis. The normal distribution of the continuous variables was tested using the Kolmogorov-Smirnov test. Independent group t-test, Mann-Whitney U test, and chi-square test were used to compare independent variables with normal distribution, independent variables without normal distribution, and categorical data, respectively. Log rank test was used to determine the BCR-free survival of both surgical methods. Kaplan-Meier analysis was performed to estimate survival rates. P-values of $<0.05$ in the 95\% confidence interval were considered significant.

\section{Results}

In total, 231 and 212 of the patients underwent RRP and RARP, respectively. The demographic characteristics of the patients were generally comparable in both surgical groups (Table 1). Only, the PSA value was lower in the RARP group (Table 1).

Intraoperative blood loss was less in the RARP group (540 $\mathrm{mL}$ vs $265 \mathrm{~mL} ; \mathrm{p}<0.001$ ) (Table 2). The mean hospitalization

\begin{tabular}{|c|c|c|c|}
\hline Variables & $\begin{array}{l}\text { RRP } \\
(n=231)\end{array}$ & $\begin{array}{l}\text { RARP } \\
(n=212)\end{array}$ & p-value* \\
\hline $\begin{array}{l}\text { Mean age at surgery } \pm S D \\
(y r)\end{array}$ & $65.54 \pm 6.57$ & $69.59 \pm 6.51$ & 0.372 \\
\hline \multicolumn{3}{|l|}{ Body mass index $\left(\mathrm{kg} / \mathrm{m}^{2}\right)$} & \multirow{3}{*}{0.874} \\
\hline$<30\left(\mathrm{~kg} / \mathrm{m}^{2}\right)$ & $171(74 \%)$ & $160(75.5 \%)$ & \\
\hline$\geq 30\left(\mathrm{~kg} / \mathrm{m}^{2}\right)$ & $60(26 \%)$ & $52(24.5 \%)$ & \\
\hline History of inguinal hernia & $21(9.1 \%)$ & $22(10.3 \%)$ & 0.614 \\
\hline History of abdominal surgery & $45(19.5 \%)$ & $46(21.6 \%)$ & 0.490 \\
\hline $\begin{array}{l}\text { Preoperative erection sufficient } \\
\text { for sexual intercourse }\end{array}$ & $153(66.2 \%)$ & $142(67 \%)$ & 0.780 \\
\hline Preoperative continent & $231(100 \%)$ & $212(100 \%)$ & - \\
\hline $\begin{array}{l}\text { Median preoperative PSA (ng/ } \\
\mathrm{mL} \text { ) (IQR) }\end{array}$ & $\begin{array}{l}13.22 \\
(5.68-17.98)\end{array}$ & $\begin{array}{l}9.30 \\
(5.19-12.5)\end{array}$ & $0.018^{*}$ \\
\hline \multicolumn{3}{|l|}{ Biopsy ISUP grade } & \multirow{4}{*}{0.112} \\
\hline ISUP grade 1 & $119(51.5 \%)$ & $121(57.1 \%)$ & \\
\hline ISUP grade 2-3 & $59(25.5 \%)$ & $54(25.5 \%)$ & \\
\hline ISUP grade 4-5 & $53(22.9 \%)$ & $37(17.4 \%)$ & \\
\hline \multicolumn{3}{|l|}{ Clinical T-stage } & \multirow{3}{*}{0.767} \\
\hline cT1 & $120(51.9 \%)$ & $109(51.4 \%)$ & \\
\hline cT2 & $111(48.1 \%)$ & $103(48.6 \%)$ & \\
\hline \multicolumn{4}{|c|}{$\begin{array}{l}\text { *Mann-Whitney U test, IQR: Interquartile range, RRP: Retropubic radical } \\
\text { prostatectomy, RARP: Robot-assisted radical prostatectomy, PSA: Prostate- } \\
\text { specific antigen, SD: Standard deviation, ISUP: International Society of Urologic } \\
\text { Pathology }\end{array}$} \\
\hline
\end{tabular}


duration was 5.62 days in the RRP group and 4.24 days in the RARP group, and the difference was significant $(p=0.046)$ (Table 2). The indwelling catheter duration was shorter in the RARP group (15.11 vs 8.75; $\mathrm{p}<0.001$ ) (Table 2 ). The modified Clavien classification system was used to evaluate postoperative complications (6). In total, 28 complications were observed, including $17(7.4 \%)$ in the RRP group and $11(5.2 \%)$ in the RARP group. The number of patients with grade 3 complications was $2(0.8 \%)$ in the RRP group and $1(0.5 \%)$ in the RARP group. Clavien 4-5 complications were not observed in any patient. The number of patients with postoperative complications was not significantly different $(p=0.224)$ (Table 2$)$.

Better continence rate was found in the RARP group at 3 months postoperatively (58.9\% vs $74.5 \% ; p=0.021$ ). Despite the higher continence rate in the RARP group at 12 months postoperatively, this difference was not significant $(79.2 \%$ vs $84.9 \%$; $p=0.398)$ (Table 3).

While the potency rates of the patients were lower at 3 months postoperatively (10.5\% vs $10.6 \%)$, the potency rates improved at 12 months postoperatively (39.2\% vs $42.3 \%$ ). Although better potency rates were recorded in the RARP group, the difference was not significant $(p=0.695)$ (Table 3$)$.

\begin{tabular}{|c|c|c|c|}
\hline Variables & $\begin{array}{l}\text { RRP } \\
(n=231)\end{array}$ & $\begin{array}{l}\text { RARP } \\
(n=212)\end{array}$ & p-value* \\
\hline \multicolumn{3}{|l|}{ Pathology ISUP grade } & \multirow{4}{*}{0.064} \\
\hline ISUP grade 1 & $101(43.7 \%)$ & $82(38.7 \%)$ & \\
\hline ISUP grade 2-3 & $84(36.4 \%)$ & $90(42.4 \%)$ & \\
\hline ISUP grade 4-5 & $46(19.9 \%)$ & $40(18.9 \%)$ & \\
\hline \multicolumn{3}{|l|}{ Pathology T-stage } & \multirow{4}{*}{0.517} \\
\hline pT2 & $184(79.7 \%)$ & $177(83.5 \%)$ & \\
\hline pT3a & $42(18.2 \%)$ & $31(14.6 \%)$ & \\
\hline pT3b & $5(2.1 \%)$ & $4(1.9 \%)$ & \\
\hline $\begin{array}{l}\text { Mean pathology prostate } \\
\text { weight } \pm \text { SD }\end{array}$ & $48.08 \pm 17.84$ & $47.92 \pm 21.09$ & 0.769 \\
\hline $\begin{array}{l}\text { Lymph node dissection } \\
\text { performed }\end{array}$ & $145(62.8 \%)$ & $126(59.4 \%)$ & 0.390 \\
\hline $\begin{array}{l}\text { Mean lymph node yield } \\
\pm S D\end{array}$ & $13.36 \pm 6.18$ & $11.19 \pm 5.79$ & $0.044^{*}$ \\
\hline Nerve sparing & $174(75.3 \%)$ & $165(77.8 \%)$ & 0.419 \\
\hline $\begin{array}{l}\text { Pathology negative surgical } \\
\text { margin }\end{array}$ & $178(77.1 \%)$ & $172(81.1 \%)$ & 0.259 \\
\hline Blood loss \pm SD $(\mathrm{mL})$ & $540 \pm 67$ & $265 \pm 41$ & $<0.001^{*}$ \\
\hline $\begin{array}{l}\text { Indwelling catheter } \pm \text { SD } \\
\text { (day) }\end{array}$ & $15.11 \pm 3.14$ & $8.75 \pm 3.05$ & $<0.001^{*}$ \\
\hline Hospitalization \pm SD (day) & $5.62 \pm 1.78$ & $4.24 \pm 1.73$ & $0.046^{*}$ \\
\hline \multicolumn{3}{|l|}{ Postoperative complication } & \multirow{5}{*}{0.224} \\
\hline Grade 1 & $10(4.3 \%)$ & $6(2.8 \%)$ & \\
\hline Grade 2 & $5(2.2 \%)$ & $4(1.9 \%)$ & \\
\hline Grade $3 a$ & $1(0.4 \%)$ & $1(0.5 \%)$ & \\
\hline Grade $3 b$ & $1(0.4 \%)$ & - & \\
\hline \multicolumn{4}{|c|}{$\begin{array}{l}\text { *Independent simple t-test; SD: Standard deviation, RRP: Retropubic radical } \\
\text { prostatectomy, RARP: Robot-assisted radical prostatectomy, ISUP: International } \\
\text { Society of Urologic Pathology }\end{array}$} \\
\hline
\end{tabular}

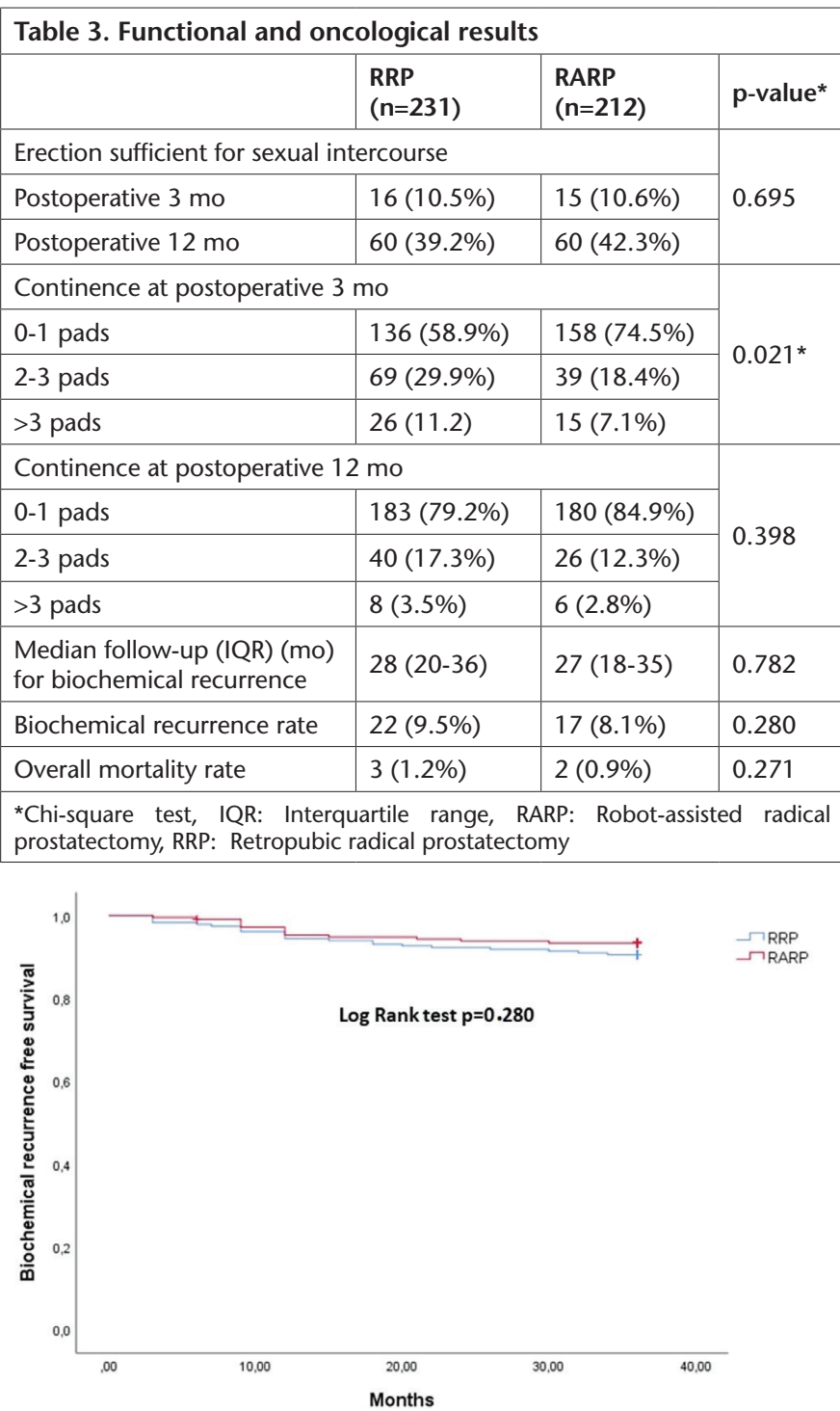

Figure 1. Kaplan-Meier curves for biochemical recurrence-free survival Biochemical recurrence-free survival was $90.5 \%$ in the RRP group versus $91.9 \%$ in the RARP group. Kaplan-Meier analysis did not show a significant difference between biochemical recurrence-free survival rates of the two groups $(p=0.280)$ RARP: Robot-assisted radical prostatectomy, RRP: Retropubic radical prostatectomy

The median follow-up for BCR was 28 (20-36) and 27 (18-35) months in the RRP and RARP groups, respectively. The mean follow-up time of both groups was comparable (Table 3 ). The BCR-free survival rate was $90.5 \%$ in the RRP group versus $91.9 \%$ in the RARP group. Kaplan-Meier analysis did not demonstrate any significant difference between BCR-free survival rates of both groups $(p=0.280)$ (Figure 1).

NS-RP was performed at comparable rates in both surgical arms (75.3\% vs 77.8\%; $p=0.419$ ) (RRP arm: unilateral NS-RP, 54; bilateral NS-RP, 120; RARP arm: unilateral NS-RP, 50; bilateral NSRP, 115). NS-RP was not performed on patients with CT3 or ISUP grade $>3$. Patients with positive surgical margins were similar in both surgical groups (22.9\% vs $18.9 \% ; p=0.259$ ) (Table 2 ). 
The rates of patients who underwent pelvic $\mathrm{LN}$ dissection (PLND) among the groups were close to each other $(75.3 \%$ vs $77.8 \%$; $\mathrm{p}=0.419$ ). The mean number of $L N$ yield was 13.36 and 11.19 in the RRP and RARP groups, respectively, and the difference was significant in favor of RRP ( $p=0.044)$ (Table 2).

\section{Discussion}

In this study, we evaluated oncological results with total PSA measurements in the postoperative follow-up. We found that the proportion of patients with BCR was higher in the RRP arm than in the RARP arm, but this difference was not significant. Some of the risk factors for BCR include pathology with ISUP grade $\geq 2$, positive surgical margin, extracapsular spread, seminal vesicle involvement, and positive LN. Providing negative surgical margin is very important for cancer control. The detection of surgical margin positivity in patients with pT2 increases the risk of BCR by $12 \%$ (7). Surgeon's experience, prostate volume, risk group of tumor, tumor volume, and degree of excision of the neurovascular bundle may affect surgical margin positivity (8). In both groups with a similar rate of NS-RP, more than $80 \%$ negative surgical margins were achieved. Despite a proportionally small difference in favor of RRP, this difference was not significant. A study compared the positive surgical margin in both surgical arms, which revealed favorable results of RARP, but the patient population was worse in the RRP arm (8). However, results of a large-scale meta-analysis on this topic are similar to our results (9). Similarly, a prospective randomized controlled study comparing RRP and RARP did not find a difference between the two groups in terms of receiving additional oncological treatments such as RT or androgen deprivation therapy (10).

In PCa, PLND is important for ensuring correct staging, providing information about prognosis, and determining adjuvant treatment needs. In addition, the number of $\mathrm{LN}$ yield in patients with positive $L N$ and cancer-specific survival was correlated (11). The probability of LN invasion in high-risk PCa is $20 \%$ (12). While LN metastasis is detected in $10-24.1 \%$ of patients with extended PLND, this rate decreases to $0-5.2 \%$ in standard PLND (13). Owing to the variable lymphatic drainage of the prostate, extended PLND is recommended for all patients with PLND indication (14). Although the rates of patients with extended PLND in our study were similar in the RRP and RARP groups, the number of LN yield was significantly higher in the RRP group. Studies with large patient populations using the Surveillance Epidemiology and End Results medicare-linked database have shown that extended PLND is less common in patients undergoing RARP $(15,16)$. These results may be due to limited movements of the robot arms, difficulty of the learning curve, and RARP performed by urologists with little experience in uro-oncology.

In studies comparing continence, the lack of standardization due to the definition of continence and differences in research makes it difficult to evaluate these results. In the literature, postprostatectomy incontinence rates were $7-39 \%$ and $4-31 \%$ in the RRP and RARP groups, respectively $(17,18)$. The wide range of these ratios was related to both the lack of a standard definition for incontinence and the surgeon's experience. According to our study, although a difference was found between the two groups for the continence rates at 3 moths postoperatively, the rates were comparable at 12 months postoperatively. Some studies have shown that it is better to report continence in the RARP group; however, in meta-analysis of long-term prospective studies, the two groups showed a similar rate of continence. $(10,18)$. Bladder neck protector technique, neurovascular bundle dissection, and prolonged urethra can be made easier in RARP with high magnification. This may explain that early continence is better in the RARP group.

In studies comparing the two surgical approaches in terms of potency, there is no standard definition for potency. While some used symptom scores for potency, such as IIEF-5, some defined potency as a coit ability. We considered patients who were able to provide adequate erections for sexual intercourse as potent. The rates of patients who were potent at the 3 and 12 months postoperatively were very comparable in both groups. Preoperatively, the rate of achieving erection sufficient for sexual intercourse in all patients who underwent RP was $66.6 \%$ (RRP group, $66.2 \%$; RARP group, 67\%). Regardless of the surgical approach, the 3- and 12-month potency rates of all patients who underwent NS-RP were 10.5 and $40.7 \%$, respectively. In other words, $59.3 \%$ of the patients who underwent NS-RP developed erectile dysfunction. This high rate may be attributed to our preference for the rate of achieving erection sufficient of sexual intercourse rather than the IIEF- 5 score when evaluating erectile function. Studies have shown that $25-75 \%$ of patients who performed RP develop erectile dysfunction later (19). A meta-analysis showed that RARP is a superior procedure than RRP in terms of the 12-month potency rates (20). However, 2-year results of a randomized controlled study reported no difference in sexual function between both surgical techniques (10). The postoperative sexual function is thought to be related to patient age, surgeon experience, and neurovascular bundle preservation $(21,22)$.

The hospitalization duration of patients who underwent RARP was shorter. In accordance with our routine practice, the catheters were removed on average in 14 days in patients with RRP and in 7 days in patients with RARP. Therefore, the duration of catheterization was shorter in the RARP group.

There was more blood loss in the RRP group than in the RARP group. Generally, blood loss is less in the RARP arm due to the buffering effect of the intra-abdominal pressure and the ability of the robot arms to be used under the good vision of the surgeon. The fact that the hospitalization duration, catheterization time, and amount of blood loss is less in the RARP arm is caused by the fact that RARP is a more minimally invasive procedure.

The total complication rates were $7.3 \%$ and $5.2 \%$ in the RRP and RARP groups, respectively. Complication rates were comparable. Different complications may occur in patients undergoing RARP depending on the patient position (Trendelenburg), intraabdominal pressure, and robotic arms, unlike RRP.

\section{Study Limitations}

This study has some limitations. This study is a retrospective study from a single center with multiple surgeons. This may cause differences between surgical indications, techniques, and managements. The short follow-up period is also another 
limitation, especially for the monitoring of oncological outcomes. Nevertheless, our functional outcomes were similar to those of previous studies $(10,23)$.

\section{Conclusion}

Although the oncological and functional results of RRP and RARP are comparable, perioperative adverse events tend to be less common in RARP because of the minimally invasive characteristics of the procedure. We believe that the surgeon's experience has larger effect than the chosen technique on oncological and functional results.

\section{Acknowledgements}

Publication: The results of the study were not published in full or in part in form of abstracts.

Contribution: There is not any contributors who may not be listed as authors.

Conflict of Interest: No conflict of interest was declared by the authors.

Financial Disclosure: The authors declared that this study received no financial support.

\section{Ethics}

Ethics Committee Approval: The local ethical committee's registration number is 13-192-20.

Informed Consent: Retrospective study.

Peer-review: Externally peer-reviewed.

\section{Authorship Contributions}

Concept: M.A.I., Design: M.A.I., S.B., K.T., Data Collection or Processing: E.K., K.T., Analysis or Interpretation: S.B., Literature Search: Ç.A., E.K., E.S., Writing: M.A.I.,

\section{References}

1. Young HH. XV. Cancer of the prostate: a clinical, pathological and post-operative analysis of 111 cases. Ann Surg 1909;50:1144.

2. Millin T. Retropubic prostatectomy a new extravesical technique: report on 20 cases. Lancet 1945;246:693-696.

3. Walsh PC, Lepor H, Eggleston JC. Radical prostatectomy with preservation of sexual function: anatomical and pathological considerations. Prostate 1983;4:473-485.

4. Abbou C C, Hoznek A, Salomon L, et al. Laparoscopic radical prostatectomy with a remote controlled robot. J Urol 2001; 165:1964-1966.

5. MOUL JW. Prostate specific antigen only progression of prostate cancer. J Urol 2000;163:1632-1642.

6. Dindo D, Demartines $N$, Clavien $P$ A. Classification of surgical complications: a new proposal with evaluation in a cohort of 6336 patients and results of a survey. Ann Surg 2004;240:205.

7. Budäus $L$, Isbarn $H$, Eichelberg $C$, et al. Biochemical recurrence after radical prostatectomy: multiplicative interaction between surgical margin status and pathological stage. J Urol 2010;184:1341-1346.
8. Suardi N, Dell'Oglio P, Gallina A, et al. Evaluation of positive surgical margins in patients undergoing robot-assisted and open radical prostatectomy according to preoperative risk groups. Urol Oncol 2016;34:57.e1-7. doi: 10.1016/j.urolonc.2015.08.019.

9. Novara G, Ficarra V, Mocellin S, et al. Systematic review and metaanalysis of studies reporting oncologic outcome after robot-assisted radical prostatectomy. Eur Urol 2012;62:382-404.

10. Coughlin GD, Yaxley JW, Chambers SK, et al. Robot-assisted laparoscopic prostatectomy versus open radical retropubic prostatectomy: 24-month outcomes from a randomised controlled study. Lancet Oncol 2018;19:1051-1060.

11. Abdollah F, Gandaglia G, Suardi N, et al. More extensive pelvic lymph node dissection improves survival in patients with node-positive prostate cancer. Eur Urol 2015;67:212-219.

12. Abdollah F, Suardi N, Gallina A, et al. Extended pelvic lymph node dissection in prostate cancer: a 20 -year audit in a single center. Ann Oncol 2013; 24:1459-1466.

13. Li R, Petros FG, Kukreja JB, et al. Current technique and results for extended pelvic lymph node dissection during robot-assisted radical prostatectomy. Invest Clin Urol 2016;57:S155-164.

14. Briganti A, Larcher A, Abdollah F, et al. Updated nomogram predicting lymph node invasion in patients with prostate cancer undergoing extended pelvic lymph node dissection: the essential importance of percentage of positive cores. Eur Urol 2012;61:480-487.

15. Schiffmann J, Larcher A, Sun M, et al. Suboptimal use of pelvic lymph node dissection: Differences in guideline adherence between robot-assisted and open radical prostatectomy. Can Urol Assoc J 2016;10:269.

16. Gandaglia G, Trinh Q-D, Hu J, et al. The impact of robot-assisted radical prostatectomy on the use and extent of pelvic lymph node dissection in the "post-dissemination" period. Eur J Surg Oncol 2014;40:1080-1086.

17. Ficarra V, Novara G, Artibani W, et al. Retropubic, laparoscopic, and robot-assisted radical prostatectomy: a systematic review and cumulative analysis of comparative studies. Eur Urol 2009;55:1037-1063.

18. Ficarra V, Novara G, Rosen RC, et al. Systematic review and metaanalysis of studies reporting urinary continence recovery after robotassisted radical prostatectomy. Eur Urol 2012;62:405-417.

19. Sanda MG, Dunn RL, Michalski J, et al. Quality of life and satisfaction with outcome among prostate-cancer survivors. N Eng J Med 2008;358:1250-1261.

20. Ficarra $V$, Novara $G$, Ahlering TE, et al. Systematic review and metaanalysis of studies reporting potency rates after robot-assisted radical prostatectomy. Eur Urol 2012;62:418-430.

21. Khoder WY, Waidelich R, Seitz M, et al. Do we need the nerve sparing radical prostatectomy techniques (intrafascial vs. interfascial) in men with erectile dysfunction? Results of a single-centre study. World I Urol 2015;33:301-307.

22. Salonia A, Adaikan G, Buvat J, et al. Sexual rehabilitation after treatment for prostate cancer-part 1: recommendations from the Fourth International Consultation for Sexual Medicine (ICSM 2015). J Sex Med 2017;14:285-296.

23. Yaxley JW, Coughlin GD, Chambers SK, et al. Robot-assisted laparoscopic prostatectomy versus open radical retropubic prostatectomy: early outcomes from a randomised controlled phase 3 study. Lancet 2016;388:1057-1066. 